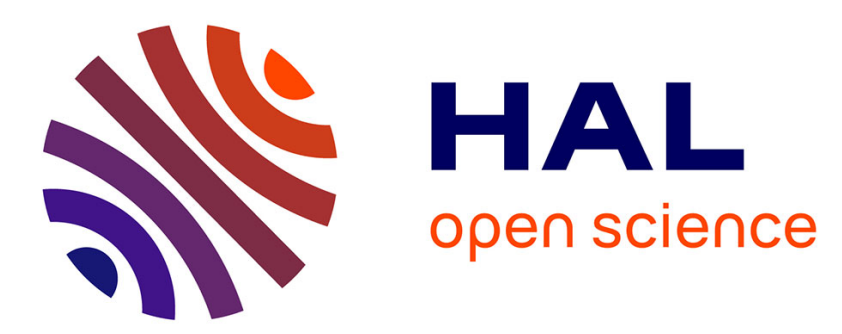

\title{
Gain scheduled control strategies for a nonlinear electrostatic microgripper: Design and real time implementation
}

Mokrane Boudaoud, Yann Gorrec, Yassine Haddab, Philippe Lutz

\section{- To cite this version:}

Mokrane Boudaoud, Yann Gorrec, Yassine Haddab, Philippe Lutz. Gain scheduled control strategies for a nonlinear electrostatic microgripper: Design and real time implementation. 51st IEEE Annual Conference on Decision and Control, CDC'12., Nov 2012, Maui - Hawaii, United States. pp.3127-3132. hal-01313540

\author{
HAL Id: hal-01313540 \\ https://hal.science/hal-01313540
}

Submitted on 10 May 2016

HAL is a multi-disciplinary open access archive for the deposit and dissemination of scientific research documents, whether they are published or not. The documents may come from teaching and research institutions in France or abroad, or from public or private research centers.
L'archive ouverte pluridisciplinaire HAL, est destinée au dépôt et à la diffusion de documents scientifiques de niveau recherche, publiés ou non, émanant des établissements d'enseignement et de recherche français ou étrangers, des laboratoires publics ou privés. 


\title{
Gain scheduled control strategies for a nonlinear electrostatic microgripper: Design and real time implementation
}

\author{
Mokrane Boudaoud, Yann Le Gorrec, Yassine Haddab and Philippe Lutz
}

\begin{abstract}
This paper deals with the accurate and fast positioning control of a nonlinear electrostatically actuated microgripper. Considering the importance of nonlinearities, performances are achieved through the design of gain scheduled controllers. To this end, a nonlinear model of the studied system is proposed and is reformulated into a polynomial LPV (Linear Parameter Varying) model. Controllers are designed considering the particular polynomial parametric dependence of the LPV model. In a first instance, a controller is synthesized using an affine LPV descriptor representation of the system and LMI (Linear Matrix Inequality) constraints. In a second instance, to deal with real time implementation constraints, a second controller is designed based on an iterative procedure using the eigenstructure assignment methodology and a worst case analysis. For embedded applications, requiring simple controller structures, we show experimentally the interest of the iterative procedure which can achieve good results relatively with the ones obtained using recent advances of robust controllers based on LMI conditions.
\end{abstract}

\section{INTRODUCTION}

Microgrippers are efficient tools for the manipulation of objects at the micrometer scale. They are widely used in microassembly [1] and biomedical research [2]. Electrostatic comb drive actuators are among the most used actuation mechanisms within microgrippers [3][4]. They allow a rapid positioning with nanometer resolutions and they have no hysteretic behavior. A comb drive actuator consists of two interdigitated finger structures, where one comb is fixed and the other is connected to a compliant mechanism. Comb dive actuators generate large displacements (several tens of $\mu \mathrm{m}$ ) but they become highly nonlinear when exceeding a threshold of displacement (few $\mu \mathrm{m}$ ). Nonlinearities arise from external electrostatic potentials [5], from axial forces acting on the clamped parts of the suspensions [6] and also from the damping which increases with increasing the displacement of the actuator [7]. A recent overview on the nonlinearities in MEMS (Micro Electro Mechanical Systems) can be found in [8]. It is therefore very difficult to ensure control performances for the micropositioning of such actuators in a wide operating range.

In the literature, some robust control strategies for comb drive actuators have been investigated (see [9] for a recent survey of MEMS control). A linear time invariant $H_{\infty}$ methodology is proposed in [10] to control the position of an electrostatic microgripper taking into account model uncertainties. A robust sliding mode strategy is designed

.The authors are with the Automatic Control and Micro Mechatronic Systems Department, FEMTO-ST Institute, UFC-ENSMM-UTBM-CNRS, University of Franche-Comté, Besançon, France. (mokrane.boudaoud, yassine.haddab, legorrec, philippe.lutz)@femto-st.fr in [7] to control a MEMS optical switch considering the damping of the comb drive actuator as the only nonlinear parameter. Direct and indirect sliding mode control [11][?], sliding mode control with an observer [12] or with an adaptive gain [13] proved that they are efficient to control comb drive actuators used in MEMS gyroscope. Most of related control techniques do not tackle the problem of positioning the actuator in a large operating range. This requirement is crucial in micromanipulation tasks for which positioning performances must be ensured regardless of the size of the manipulated object. According to author's knowledge, such an issue has not been analyzed previously and especially in the case of MEMS based microgrippers.

The aim of this paper is the control of electrostatic type microgrippers using nonlinear comb drive actuators. A microgripper (FT-G100) from FemtoTools GmbH Company [3] is used as a case of study. Controllers are designed to ensure some performance level over a wide operating range despite nonlinearities. Therefore, we propose the design of gain scheduled controllers to achieve required specifications. Nonlinearities induce large variations of the stiffness and the damping of the system. Instead of using the stiffness and the damping "independently" as scheduling variables, we propose to use the position of the gripper arm as the only variant parameter. To do so, a Linear Parameter Varying (LPV) model of the actuation mechanism with a polynomial parametric dependence is proposed. This polynomial dependence causes some issues for the derivation of the controller. As such, two gain scheduled control strategies are proposed: the first one uses an affine LPV descriptor model and a finite number of linear matrix inequalities (LMIs) to derive the controller and the second one consists on a robust eigenstructure assignment. The paper is organized as follows. In section II, global architecture of the FT-G100 microgripper is presented. The third section is devoted to the nonlinear and the LPV modeling of the system. Control strategies are presented in the fourth section. In section V, experimental control results are presented. Conclusions end the paper.

\section{GLOBAL ARCHITECTURE OF THE MICROGRIPPER}

The FT-G100 microgripper is designed to handle objects ranging from $1 \mu \mathrm{m}$ to $100 \mu \mathrm{m}$. The initial opening of the gripper arms is $100 \mu \mathrm{m}$. The microgripper consists of two distinct parts (see Fig. 1): -an actuation mechanism composed of a comb drive actuator and an actuated arm, -a sensing mechanism including a capacitive force sensor and a sensing arm. The latter is not studied in this paper. The base of the actuated arm is attached to a flexure joint. Furthermore 
a suspension mechanism including two pairs of clampedclamped beams holds the movable part of the actuator.

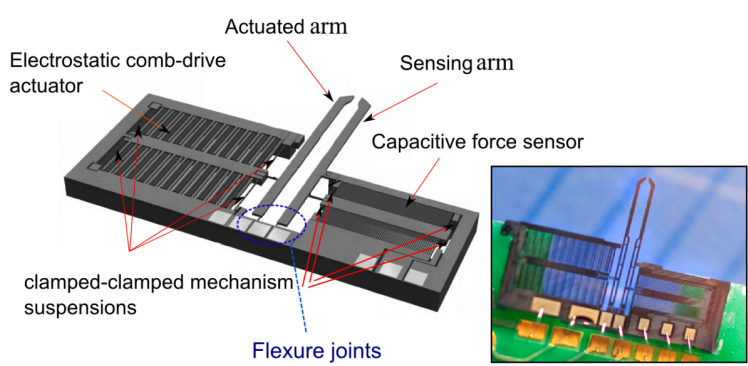

Fig. 1. Structure of the FT-G100 microgripper (FemtoTools GmbH).

\section{MOdELING OF THE ACTUATION MECHANisM}

\section{A. Nonlinear dynamic modeling}

The dynamic model of the actuation mechanism is based on the nonlinear Duffing equation which takes into account axial forces $\vec{N}$ acting on the clamped parts of the suspensions (Fig. 2a). Such forces are considered by introducing a cubic stiffness $k_{a 3}$ in the dynamic equation [8]. In the case of the microgripper, the Duffing equation is given as:

$$
m_{a} \cdot \frac{d^{2} y_{a}(L)}{d t^{2}}+d_{a} \cdot \frac{d y_{a}(L)}{d t}+k_{a 1} \cdot y_{a}(L)+k_{a 3} \cdot y_{a}^{3}(L)=\frac{1}{D_{a}} \cdot F_{\text {elec }}
$$

$m_{a}, d_{a}$ and $k_{a 1}$ are respectivelythe linear mass, the linear damping and the linear stiffness of the actuation mechanisms, $D_{a}$ is an amplification factor.
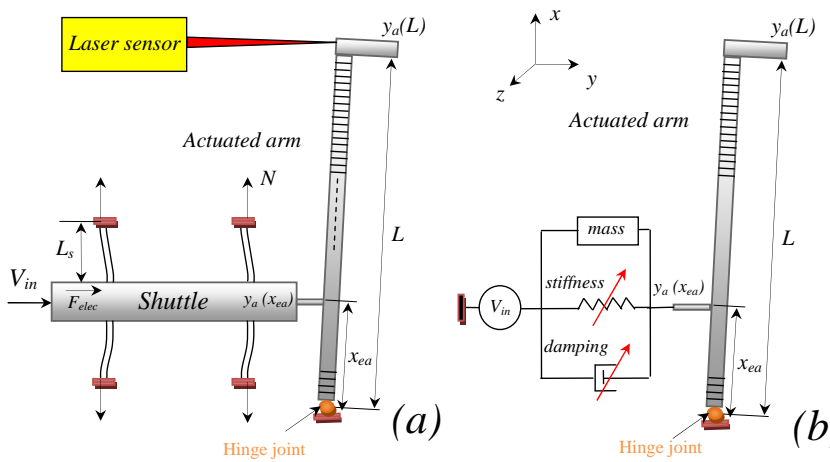

Fig. 2. Simplified scheme of the FT-G100 actuation mechanism (a) and equivalent scheme of suspension mechanism (b).

The electrostatic force $F_{\text {elec }}$ generated by the comb drive actuator is defined as:

$$
F_{\text {elec }}=\frac{N_{a} \cdot \varepsilon \cdot h_{z}}{2 \cdot g} \cdot V_{\text {in }}^{2}
$$

Where, $N_{a}=1300$ is the number of comb fingers, $\varepsilon=$ $8.85 \mathrm{pF} / \mathrm{m}$ is the permittivity of the air, $h_{z}=50 \mu \mathrm{m}$ is the thickness of comb fingers, and $g=6 \mu \mathrm{m}$ is the gap spacing between two fingers. Under the assumption that the base of the actuated arm behaves as a hinge joint (Fig. 2) and that the actuated arm is rigid, the amplification mechanism is given as: $D_{a}=\frac{L}{x_{e a}}$, with $L=5150 \mu \mathrm{m}$ and $x_{e a}=1100 \mu \mathrm{m}$.
A laser interferometer sensor (SP-120 SIOS Metechnik $\mathrm{GmbH}$ ) is used to derive the experimental static characteristic $y_{a}(L) / V_{\text {in }}$ from 0 to $200 \mathrm{~V}$. Experimental data are then fitted using equation (1) in static mode. With a third order polynomial interpolation, the mean fitting of the static characteristic is found to be equal to $17.11 \%$. To reduce this error, we have extended the Duffing equation by considering a sixth order polynomial in the static characteristic leading to the following expression: $\sum_{i=1}^{6} k_{a i} \cdot y_{a}^{i}(L)=\frac{N_{a} \cdot \varepsilon \cdot h_{z}}{2 \cdot g \cdot D_{a}} \cdot V_{i n}^{2}$. This polynomial extension allows taking into account additional nonlinearities that are not considered in the standard Duffing equation. Therefore, by fitting experimental data with the extended Duffing static equation, the mean error is reduced to $3.66 \%$ and results are presented in Fig. 3a.


Fig. 3. Nonlinear characteristics of the FT-G100 actuation mechanism (a) static actuated arm tip displacement/ supply voltage, (b) stiffness/actuated arm tip displacement, (c) damping/actuated arm tip displacement.

Identified parameters are given as: $k_{\mathrm{a} 1}=1,0762 \mathrm{~N} / \mathrm{m}$, $k_{\mathrm{a} 2}=-1,6 \times 10^{3} \mathrm{~N} / \mathrm{m}^{2}, k_{\mathrm{a} 3}=4,88 \times 10^{8} \mathrm{~N} / \mathrm{m}^{3}, k_{\mathrm{a} 4}=-1,69 \times$ $10^{12} \mathrm{~N} / \mathrm{m}^{4}, k_{\mathrm{a} 5}=-3,98 \times 10^{16} \mathrm{~N} / \mathrm{m}^{5}, k_{\mathrm{a} 6}=4,71 \times 10^{20} \mathrm{~N} / \mathrm{m}^{6}$. Consequently, the nonlinear evolution of the stiffness in the actuation mechanism is deduced as shown in Fig. 3b.

To identify the mass and the damping, step excitations are applied to the actuator in the operating range 0 to $200 \mathrm{~V}$ and the displacements $y_{a}(L)$ are measured with the laser sensor. It is observed that the damping of the system increases with increasing the amplitude of motion starting from $y_{a}(L)=60 \mu \mathrm{m}$. A nonlinear damping term of the form $d_{a}\left(y_{a}(L)\right)=\sum_{i=0}^{4} d_{a i} \cdot y_{a}^{i}(L)$ is then introduced in (1). A fourth order polynomial has been sufficient to describe the nonlinear damping (Fig. 3c). The mass is identified with the experimental step response of the actuation mechanism for $5 \mathrm{~V}$ input voltage. Moreover, the damping is identified at each operating point from experimental step responses and results are fitted on the fourth order polynomial. Identified parameters are: $m_{a}=3,9843 \times 10^{-8} \mathrm{Kg}, d_{\mathrm{a} 0}=3,01 \times 10^{-6} N s / m, d_{\mathrm{a} 1}=$ $-0,162 N s / m^{2}, d_{\mathrm{a} 2}=1,24 \times 10^{4} N s / m^{3}, d_{\mathrm{a} 3}=-3,04 \times 10^{8} N s / m^{4}$, $d_{\mathrm{a} 4}=2,47 \times 10^{12} \mathrm{Ns} / \mathrm{m}^{5}$. 
Taking into acoount the nonlinear stiffness and damping, we obtain the following nonlinear state space model:

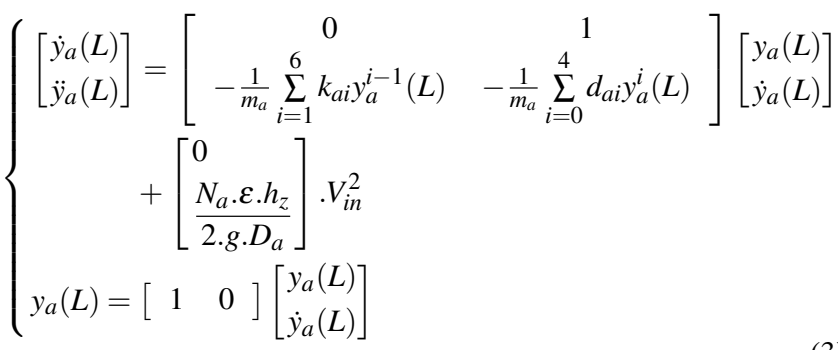

For the model validation, the experimental frequency spectrum of the system is compared with the simulated one using (3) and a good agreement is observed Fig. 4. The first resonance frequency varies from $827 \mathrm{~Hz}$ to about $2 \mathrm{KHz}$. Moreover three vibration modes appear staring from $V_{\text {in }}=50$ V. Such characteristics are well captured in the nonlinear model. For control purposes, the model (3) is reformulated into a LPV model in such a way that the position of the actuated arm tip is the only variant parameter.
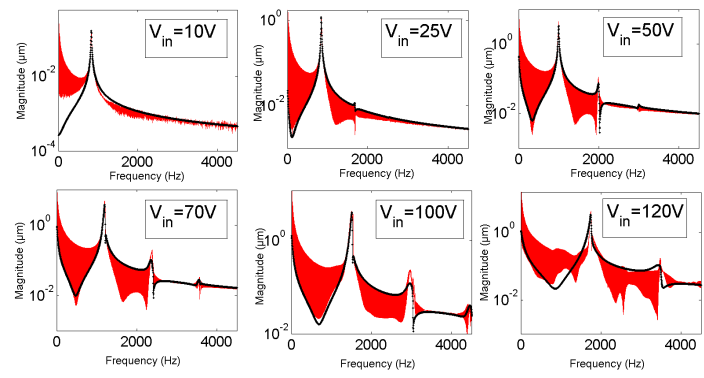

Fig. 4. Frequency responses of the nonlinear actuation mechanism: experimental data (red curve) and simulation data (black curve)

\section{B. LPV modeling}

The nonlinear model (3) can be reformulated into an affine LPV model if the damping and the stiffness are chosen independently as varying parameters Fig. 2b. For this class of LPV models, well known gain scheduled control strategies such as LPV/H$H_{\infty}[14]$ can be used. The main drawback of this approach is that the polynomial dependence of the nonlinear parameters is not taken into account during the controller design leading to a high conservatism. In the present work, we propose to use the operating point (actually the position of the actuated arm tip) as the only varying parameter. To this end, using a Jacobian linearization, the nonlinear plant (3) is formulated into a polynomial LPV model of the form:

$$
\begin{aligned}
& \left\{\begin{array}{l}
\dot{X}_{p}(t)=A_{p}(\delta) X_{p}(t)+B_{p} \cdot U(t) \\
\tilde{y}_{a}(L, t)=C_{p} X_{p}(t)
\end{array}\right. \\
& A_{p}(\boldsymbol{\delta})=\left[\begin{array}{cc}
0 & 1 \\
-\frac{1}{m_{a}} \cdot \sum_{i=1}^{6} i \cdot k_{a i} \cdot \delta^{i-1} & -\frac{1}{m_{a}} \cdot \sum_{i=0}^{4} d_{a i} \cdot \delta^{i}
\end{array}\right] \\
& B_{p}=\left[\begin{array}{ll}
0 & \frac{N_{a} \cdot \varepsilon \cdot h_{z}}{2 \cdot g \cdot D_{a}}
\end{array}\right]^{T}, C_{p}=\left[\begin{array}{ll}
1 & 0
\end{array}\right], X_{p}=\left[\begin{array}{c}
\tilde{y}_{a}(L) \\
\dot{y}_{a}(L)
\end{array}\right] \\
& A_{p} \in R^{n \times n}, B_{p} \in R^{n \times m}, C_{p} \in R^{p \times n} \text { with } n=2, p=m=1 \text {. }
\end{aligned}
$$

$\tilde{y}_{a}(L)$ is the variation of $y_{a}(L)$ around an operating point $\delta$. To simplify notations, the variable $\tilde{y}_{a}(L)$ will be designed later by $y_{a}$. Note that, the nonlinearity arising from square input voltage is overcome by considering the variable $U=V_{\text {in }}^{2}$ as the model input in the control design.

The polynomial parametric dependence of the LPV model (4) causes some issues for the derivation of a gain scheduled controller. To overcome this control design issue, two strategies are suggested in the next section.

\section{GAIN SCHEDULED CONTROL STRATEGIES}

To design a parameter dependant controller for which the scheduled parameter is the operating point $\delta$, two distinct approaches are proposed: 1) To transform the polynomial LPV model into an affine LPV descriptor model and to design a controller with the resolution of a finite number of LMIs. 2) To perform an iterative procedure based on a worst case analysis and an eigenstructure assignment using observed based scheduled controller. Both methods finally allow designing a parameter dependant controller taking into account the particular polynomial form of (4).

\section{A. Affine LPV descriptor representation and gain scheduled control synthesis}

For the derivation of an affine LPV descriptor model of the system, starting from (4), we have performed a linear fractional transformation Fig. 5 of the form:

$$
\left[\begin{array}{l}
\dot{X}_{p} \\
y_{a} \\
z
\end{array}\right]=\left[\begin{array}{lll}
N_{11} & N_{12} & N_{13} \\
N_{21} & N_{22} & N_{23} \\
N_{31} & N_{32} & N_{33}
\end{array}\right]\left[\begin{array}{l}
X_{p} \\
U \\
v
\end{array}\right]
$$

With: $v(t)=\delta z(t), z(t) \in R^{n_{z}}, v(t) \in R^{n_{v}}$.

The sub-matrices $\left\{N_{i, j}\right\}_{i, j=1, \ldots, 3}$ are deduced from the interconnexion of the LFT. Taking into account the stiffness and damping polynomial orders, we have selected $n_{z}=n_{v}=7$ (e.i. order of the highest polynomial +1 ).

The affine LPV model (6) is obtained by defining an augmented state vector $X_{d e s}=\left[\begin{array}{cc}X_{p}^{T} & v^{T}\end{array}\right]^{T}$ such as:

$$
\begin{gathered}
\left\{\begin{array}{l}
E \dot{X}_{d e s}=A_{\text {des }}(\boldsymbol{\delta}) X_{\text {des }}+B_{\text {des }} U \\
y_{a}=C_{\text {des }} X_{\text {des }}
\end{array}\right. \\
A_{\text {des }}=\left[\begin{array}{cc}
N_{11} & N_{13} \\
\delta N_{31} & \delta N_{33}+I_{n_{v}}
\end{array}\right] \quad B_{\text {des }}=\left[\begin{array}{l}
N_{12} \\
\delta N_{32}
\end{array}\right] \\
C_{d e s}=\left[\begin{array}{lll}
C_{p} & 0_{1 \times n_{v}}
\end{array}\right] \quad E=\operatorname{diag}\left(I_{n}, 0_{n_{v}}\right)
\end{gathered}
$$

This descriptor representation is used only to derive the gain scheduled controller via the resolution of a finite number of LMIs conditions. Control performances required in this study are derived from general need in micromanipulation: no overshoot, maximum static error lower than $0.05 \%$. Moreover, we desire obtain a maximal response time lower than $30 \mathrm{~ms}$. Therefore, we have defined a reference closed loop transfer function $T_{d}$ :

$$
T_{d}=\frac{0.9995}{4 \times 10^{-6} s^{2}+0.006 \mathrm{~s}+1}
$$

The reference model $T_{d}$ contains two eigenvalues $\lambda_{1}=$ -191 and $\lambda_{2}=-1300$. In order to design the gain scheduled 
controller that ensure desired performances in wide operating range, a weighting transfer function $W_{1}(s)$ is introduced.

This function will allow tracking performances by applying specifications to the sensitivity function of the system considering a frozen value of $\delta$. In this study we have selected the frozen value as the steady tip displacement of the actuated arm for $5 \mathrm{~V}$ input voltage. The weighting function is given by $W_{1}(s)=\frac{1}{1-T_{d}}$. Therefore, the descriptor model (6) is augmented with the weighting function leading to:

$$
\left\{\begin{array}{l}
E_{g} \dot{X}_{g}=A_{g}(\delta) X_{g}+B_{1} w+B_{2} U \\
z=C_{1} X_{g}+D_{11} w \\
y_{a}=C_{2} X_{g}+D_{21} w
\end{array}\right.
$$

The external input $w=y_{a r}$ is the trajectory reference and the external output $z_{1}$ is the output of $W_{1}(s)$.

The control problem consists in designing a gain scheduled controller $K(s, \delta)$ that minimize a positive scalar $\gamma$ such as the closed loop transfer $H_{\infty}$ norm of the performance channel $w \rightarrow z_{1}$ is minimized (ideally less or equal to 1 ) for any value of the operating point $\delta$. Such requirement can be satisfied by solving the following LMI constraints [15]:

$$
\begin{gathered}
\\
{\left[\begin{array}{cc}
Y E_{g}^{T} & E_{g} \\
E_{g}^{T} & E_{g}^{T} \chi
\end{array}\right]=\left[\begin{array}{cc}
Y E_{g}^{T} & E_{g} \\
E_{g}^{T} & E_{g}^{T} \chi
\end{array}\right]^{T} \geqslant 0} \\
{\left[\begin{array}{ccc}
M_{A}+M_{A}^{T} & M_{B} & M_{C}^{T} \\
M_{B}^{T} & -\gamma I & M_{D}^{T} \\
M_{C} & M_{D} & -\gamma I
\end{array}\right]<0} \\
M_{A}=\left[\begin{array}{cc}
A_{g}(\delta) Y^{T}+B_{2} F^{T} & A_{g}(\delta) \\
H^{T} & \chi^{T} A_{g}(\delta)+G^{T} C_{2}
\end{array}\right] \\
M_{B}=\left[\begin{array}{l}
B_{1} \\
\chi^{T} B_{1}+G^{T} D_{21}
\end{array}\right] \quad M_{c}=\left[\begin{array}{ll}
C_{1} Y^{T}+D_{12} F^{T} & C_{1}
\end{array}\right]
\end{gathered}
$$

The decision variables of (9) are the matrices $\chi, Y, F, G$ and $H$ with a appropriate dimensions.

As, the varying parameter is bounded between upper $\left(\delta_{M}\right)$ and lower $\left(\delta_{m}\right)$ values, the LMI problem results in an infinite set of LMIs to solve. Nevertheless, since the matrix $A_{g}(\delta)$ is affine w.r.t the varying parameter, the LMIs (9) can be solved only on the vertices [16] of the set $\left[\delta_{m} \delta_{M}\right.$ ]. Here only two sets of LMIs must be solved since only one varying parameter is considered. If this is feasible, the solution leads to the following polytopic state space controller structure:

$$
K(s, \delta)=\alpha_{1}(\delta)\left[\begin{array}{cc}
A_{k 1} & B_{k 1} \\
C_{k 1} & 0
\end{array}\right]+\alpha_{2}(\delta)\left[\begin{array}{cc}
A_{k 2} & B_{k 2} \\
C_{k 2} & 0
\end{array}\right]
$$

The matrices $\left\{A_{k i}, B_{k i}, C_{k i}\right\}$ are derived from the solution of the LMIs (9) and $\left\{\alpha_{1}(\delta), \alpha_{2}(\delta)\right\}$ are given by $\alpha_{1}(\delta)=\frac{\delta_{M}-\delta}{\delta_{M}-\delta_{m}}$ and $\alpha_{2}(\delta)=1-\alpha_{1}(\delta)$.

The augmented LPV descriptor model (8), is transformed into a polytopic LPV model with two vertices. Thereafter, the LMIs conditions (9) are solved at each vertex of the model using the Matlab LMI Control Toolbox. For the resolution, we have considered $\delta_{m}=1 \mu m$ and $\delta_{M}=90 \mu m$. Consequently, two four order controllers $G_{k 1}\left\{A_{k 1}, B_{k 1}, C_{k 1}, 0\right\}$ and $G_{k 2}\left\{A_{k 2}, B_{k 2}, C_{k 2}, 0\right\}$ are obtained. Experimental control results using this strategy are presented in section V. Nevertheless, due to the order of the obtained controller, some real time implementations constraints have been encountered. The second proposed control strategy aim at overcoming this constraint while keeping desired performances.



Fig. 5. Control scheme with the augmented LPV descriptor model.

\section{B. Gain scheduled control by eigenstructure assignment}

The strategy proposed in this section is based on the output feedback control by eigenstructure assignment and multi model self scheduled control design [18]. It is well known that with the traditional eigenstructure assignment [17], the maximal number of eigenvalues that can be assigned is limited by the number of outputs of the controlled system. First, let us consider the LPV polynomial model (4) for a given frozen value $\delta_{0}$. This model has one output and the reference model contains two eigenvalues. Therefore to assign the eigenvalues of the frozen parameter system considering the reference model (7), we propose to add an observer to the polynomial LPV model based on Lemma 1.

Lemma 1: the system defined by (see Fig. 6 )

$$
\left\{\begin{array}{l}
\dot{z}_{o}=\pi_{o} z_{o}-t_{o} y_{a}+u_{o} B_{p} U \\
u_{o} A_{p}\left(\delta_{0}\right)+t_{o} C_{p}=\pi_{o} u_{o}
\end{array}\right.
$$

$u_{o} \in C^{n}, t_{o} \in C^{p}, \pi_{o} \in C$

is an observer of the variable $z_{o}=\pi_{o} X_{p}$ and the observation error $\varepsilon_{o}=z_{o}-u_{o} X_{p}$ satisfies: $\dot{\varepsilon}_{o}=\pi_{o} \varepsilon_{o}$ (see [19]). Note that $\delta_{0}$ is a frozen value of the varying parameter $\delta$.

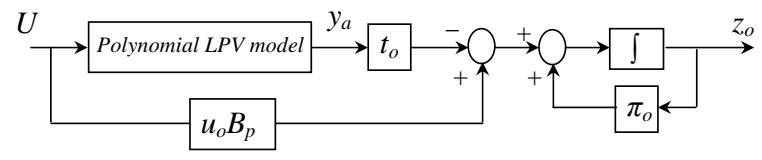

Fig. 6. Elementary observer of $z_{o}=\pi_{o} X_{p}$

Designing an output feedback controller by eigenstructure assignment to the frozen polynomial LPV model (4) with the observer consists on finding the matrix $K_{c}=\left[\begin{array}{ll}K_{y} & K_{z}\end{array}\right]$ such as the system:

$$
\left\{\begin{array}{l}
\dot{X}_{p}=A_{p}\left(\delta_{0}\right) X_{p}+B_{p} U \\
\dot{z}_{o}=\pi_{o} z_{o}+u_{o} B_{p} U-t_{o} y_{a} \\
y_{a}=C_{p} X_{p}
\end{array}\right.
$$

controlled by $U=K_{y} y_{a}+K_{z} z_{o}$ have the expected performances. This problem can be resolved by considering the separation principle described in the theorem 1 [20]. 
Theorem 1: It is equivalent to assign (by the static feedback $\left.K_{c}=\left[\begin{array}{ll}K_{y} & K_{z}\end{array}\right]\right)$ the eigenvalues of the system (12) and the one of the system (13).

$$
\left\{\begin{array}{l}
\dot{X}_{p}=A_{p}\left(\delta_{0}\right) X_{p}+B_{p} U \\
{\left[\begin{array}{l}
y_{a} \\
z_{o}
\end{array}\right]=C_{p o} X_{p}}
\end{array}\right.
$$

Where: $C_{p o}=\left[\begin{array}{ll}C_{p}^{T} & u_{o}^{T}\end{array}\right]^{T}$

The gains $K_{y}$ and $K_{z}$ allow the assignment of the eigenvalues $\lambda_{1}$ and $\lambda_{2}$ respectively. On the other hand, to ensure a statistic error lower than $0.05 \%$, we have added an integrator on the signal $y_{a r}-y_{a}$ (recall that $y_{a r}$ is the reference trajectory). In this case, the matrix $K_{c}$ is extended to $K_{c}=\left[\begin{array}{lll}K_{y} & K_{z} & K_{i}\end{array}\right]$ and the control low becomes $U=K_{i} \int \varepsilon-K_{y} y_{a}-K_{z} z_{o}$, where $\varepsilon=y_{a r}-y_{a}$. The gain $K_{i}$ is used to assign a third eigenvalue.



Fig. 7. Pole maps obtained during the iteration procedure: fist (a), second (b) and third (c) iteration.

Let us now consider the variations of the parameter $\delta$ in the set $\left[\delta_{m} \delta_{M}\right]$. In this case, the separation principle is no more satisfied $\forall \delta$. To overcome this drawback, one can modify the observer structure by choosing $u_{o}(\delta)=$ $t_{o} C_{p}\left[\pi_{o} I-A p(\delta)\right]^{-1}$ instead of $u_{o}$. As such, the separation principle is satisfied provided that the value of $\pi_{o}$ is different from any eigenvalue of the matrix $A_{p}(\delta)$. Afterward, we proceed to a multi model eigenstructure assignment. For that purpose, we increase the number of degree of freedom by choosing a posteriori a gain scheduled output feedback matrix $K_{c}(\delta)$ by mean of a second order polynomial:

$$
K_{c}(\delta)=K_{c 0}+K_{c 1} \delta+K_{c 2} \delta^{2}
$$

Thus, we apply the following iterative procedure to take into account the variation of $\delta$ :

Step 1: designing an output feedback controller with the observer on a nominal model (called $G_{n}$ ) derived from (13) using the eigenstructure assignment methodology. At this step any frozen value of the varying parameter $\delta$ can be used (this frozen value is designed as $\delta_{n}$ ).

Step 2: proceeding to a closed loop multi-model analysis of the pole map considering a finite number of operating points in the set $\left[\delta_{m} \delta_{M}\right]$. If the design is satisfactory for all the selected operating points, then stop. Otherwise identify the worst-case model (called $G_{w c}$ ), determine its critical triple $\Gamma_{i}^{*}=\left(\lambda_{i}^{*}, v_{i}^{*}, \omega_{i}^{*}\right)$ and continue with Step 3.

Step 3: improving the behavior of the worst-case model $G_{w c}$ by replacing $\Gamma_{i}$ by $\Gamma_{i}^{*}$ respecting the specifications while preserving the properties of all the models $G_{n}, \ldots, G_{w c-1}$ treated before. Return to Step 2.

The synthesis of the controller is done with respect to the couples $\left(v_{i}, \omega_{i}\right), i=0, \ldots, n_{w c}$, where $n_{w c}$ is the number of worst case models identified from the iterative procedure. Finally, taking into account the structure (14), the controller $K_{c}(\boldsymbol{\delta})$ is given such as:

$$
\left[\begin{array}{l}
K_{c 0}^{T} \\
K_{c 1}^{T} \\
K_{c 2}^{T}
\end{array}\right]^{T}=\left[\begin{array}{l}
\omega_{0}^{T} \\
\vdots \\
\omega_{n_{w c}}^{T}
\end{array}\right]^{T}\left[\begin{array}{ccc}
C_{p o}\left(\delta_{n}\right) v_{0} & \ldots & C_{p o}\left(\delta_{n_{w c}}\right) v_{n_{w c}} \\
\delta_{n} C_{p o}\left(\delta_{n}\right) v_{0} & \ldots & \delta_{n_{w c}} C_{p o}\left(\delta_{n_{w c}}\right) v_{n_{w c}} \\
\delta_{n}^{2} C_{p o}\left(\delta_{n}\right) v_{0} & \ldots & \delta_{n_{w c}}^{2} C_{p o}\left(\delta_{n_{w c}}\right) v_{n_{w c}}
\end{array}\right]^{-1}
$$

To ensure the matrix inversion in (15) two solutions are possible: performing a Moore Penrose pseudo inverse or increasing the order of the polynomial (14) at each iteration during the controller design.

For the controller synthesis, we have selected a set of 24 elementary models in the interval $\left[V_{i n}=5 \mathrm{~V} \ldots V_{\text {in }}=120 \mathrm{~V}\right]$ and 50 elementary models in the interval $\left[V_{\text {in }}=125 \mathrm{~V} \ldots V_{\text {in }}=\right.$ $185 \mathrm{~V}]$. Each interval is divided equally and from Fig. 3a the frozen value of $\delta$ related to each elementary model has been defined. We have selected more models in $\left[V_{i n}=125 \mathrm{~V} \ldots V_{\text {in }}=\right.$ $185 \mathrm{~V}]$ due to the rapid variations of the damping when the position of the actuated arm tip exceeds $60 \mu \mathrm{m}$ (see Fig. 3c). The selected nominal model corresponds to $V_{i n}=5 \mathrm{~V}$. As a result, three iterations have been required to satisfy desired performances. Identified worst case models corresponds to $V_{\text {in }}=55 \mathrm{~V}$ and $V_{\text {in }}=150 \mathrm{~V}$. The pole map obtained at each iteration is presented in Fig. 7.

The controller (14) is then deduced from (15) considering the couples $\left(v_{i}, \omega_{i}\right)$ obtained at each iteration. Obtained matrices $K_{c 0}, K_{c 1}$ and $K_{c 2}$ have only 1 line and 3 columns leading to a very simple controller structure.

\section{SySTEM SETUP AND CONTROL IMPLEMENTATION}

The system setup is composed of: the FT-G100 microgripper, the interferometer sensor, the dSPACE control board, an amplifier and a vibration isolation table where the gripper and the laser senor are fixed. Each controller is designed using the Matlab/Simulink software and a real time implementation is performed using the dSPACE control board. The displacements at the tip of the actuated arm, measured in real time by the laser sensor, are used to schedule the controllers. The root square output of the controllers is translated into an analog signal to drive the electrostatic actuator.

To emphasis the importance of the proposed gain scheduled controllers, a $L T I / H_{\infty}$ controller is designed using the model (4), for a frozen value of $\delta=\delta_{m}$, with the weighting function of Fig. 5. Therefore, for each controller, 16 step excitation references have been applied to the input of the 



Fig. 8. Experimental step responses of the controlled microgripper at different operating points (from $5 \mu \mathrm{m}$ to $90 \mu \mathrm{m}$ actuated arm tip displacement). The black curve refer to the step response of the reference model $T_{d}$. Controllers are defined as: a) method $1, \mathrm{~b}$ ) method 2, c) method 3

closed loop system. The amplitude of the step excitations varies from $5 \mu \mathrm{m}$ to $90 \mu \mathrm{m}$ with a step of $5 \mu \mathrm{m}$. Results of the controlled displacements normalized to unity (divided by the input reference) of the actuated arm tip in response to the step excitations are presented in (8). We have referred to method 1 , method 2 and method 3, the $L T I / H_{\infty}$ controller, the controller obtained by the descriptor model, and the controller based on the iterative procedure respectively.

It is clear from Fig. 8 that the $L T I / H_{\infty}$ controller cannot guarantee the required performances when performing large displacements of the actuator. Performances are respected over the operating range $5 \mu \mathrm{m}<\delta<90 \mu \mathrm{m}$ with the method 2 and the method 3 . The latter allows obtaining the desired response time, no overshoot is observed and the static error is less than $0.05 \%$. This demonstrated and justify the proposed gain scheduled controllers w.r.t the parameter $\delta$ for the control of the comb drive actuator for large displacements.

Some real time implementation constraints have been encontourned for the controller based on the methods 2. Due to the high order of the controller (two transfer functions with a fourth order denominator), the sampling frequency of the dSPACE control board had to be reduced to $15 \mathrm{KHz}$ for an efficient implementation while with the very simple structure of the controller based on method 3, the implementation could be performed easily until $100 \mathrm{KHz}$ sampling frequency. This demonstrates the interest of the method 3 for real time implementations constraints. Such a requirement is more and more needed for embedded applications in the field of robotics micromanipulation. A solution to use the method 2 for embedded application is to reduce the order of the controller. Nevertheless, controller reductions can lead to a loss of performances.

\section{CONCLUSIONS}

This paper has dealt with the gain scheduled control of a nonlinear electrostatic microgripper. A nonlinear dynamic model of the actuation mechanisms has been proposed and main nonlinear parameters have been identified experimentally. For control purposes, the nonlinear model is reformulated into a polynomial LPV model. To deal with the particular structure of the LPV model, two gains scheduled control strategies are proposed and experimental implementation results are presented. The interest of the iterative procedure for the control of comb drive actuators over large displacements in embedded applications is demonstrated with experimental arguments. The latter can achieve good results relatively with the ones obtained using recent advances of robust controllers based on LMIs conditions. Future works will concern the implementation of the controller based on the iterative procedure in micro-calculators such as FPGA. Therefore, high control performances can be achieved during a micromanipulation processes without the use of a high performance controller board such as dSPACE.

\section{REFERENCES}

[1] B. Tamadazte, E. Marchand, S. Dembele, N Le Fort-Piat, "CAD model based tracking and 3D visual-based control for MEMS microassembly", Int J of Robotics Research, vol. 29, pp. 1416-1437, 2010.

[2] X Y. Liu, K Y. Kim, Y. Zhang, Y. Sun, "Nanonewton force sensing and control in microrobotic cell manipulation", Int J of Robotics Research, vol.28, pp.1065-1076, 2009.

[3] F. Beyeler, A. Neild, S. Oberti, D. Bell, S. Yu, J. Dual, B. Nelson, "Monolithically fabricated microgripper with integrated force sensor for manipulating microobjects and biological cells aligned in an ultrasonic field", JMEMS, vol.16, pp.7-15, 2007.

[4] K. Kim, L. Xinyu, Z. Yong, Y. Sun, "Nanonewton force-controlled manipulation of biological cells using a monolithic MEMS microgripper with two-axis force feedback", J. Micromech. Microeng, vol.8, 2008.

[5] K B. Lee, A P. Pisano, L. Lin, "Nonlinear behaviors of a comb drive actuator under electrically induced tensile and compressive stresses", J.M.M, vol.17, pp.55756, 2007.

[6] R. Legtenberg, A. Groeneveld, M. Elwenspoek, "Combdrive actuators for large displacements", J.M.M, vol.6, pp.320-329,1996.

[7] B. Ebrahimi, M. Bahrami, "Robust sliding-mode control of a MEMS optical switch", Journal of Physics, vol.34, pp.728733, 2006.

[8] R. Lifshitz, M C. Cross, "Nonlinear Dynamics of Nanomechanical and Micromechanical Resonators", Review of Nonlinear Dynamics and Complexity, Edited by Heinz Georg Schuster, Wiley, 2008.

[9] A. Ferreira, S S. Aphale, "A Survey of Modeling and Control Techniques for Micro- and Nanoelectromechanical Systems", IEEE Transactions on Systems, Man, and Cybernetics, Part C: Applications and Reviews, vol.41, pp.350-364, 2011.

[10] Y. Haddab, B. Uccheddu, "Commande robuste d'une pince microfabrique actionnement lectrostatique", Conference Internationale Francophone d'Automatique CIFA 2010, Bucarest, Romania, 2008. (in French).

[11] A. Kuzu, "Control Strategies for Increased Reliability in MEM Comb Drives", Proceedings of the 2nd WSEAS Int. Conference on Applied and Theoretical Mechanics, Venice, Italy, 2006.

[12] J. Fei, C. Batur, "Adaptive sliding mode control with sliding mode observer design for a MEMS vibratory gyroscope", ASME International Mechanical Engineering Congress and Exposition, 2007.

[13] J. Fei, C. Batur, "A class of adaptive sliding mode controller with proportional-integral sliding surface", journal of system and control engeneering, vol.223, pp.989-999, 2009.

[14] A. Zin, O. Sename, P. Gaspar, L. Dugard, J. Bokor, "An LPV/Hinf Active Suspension Control for Global Chassis Technology: Design and Performance Analysis", IEEE American Control Conference, Minneapolis, USA, 2006 
[15] I. Masubuchi, J. Kato, M. Saeki, A. Ohara A, "Gain-scheduled controller design based on descriptor representation of LPV systems: Application to flight vehicle control", IEEE Conference on Decision and Control, Atlantis, Bahamas, 2004.

[16] C. Poussot-Vassal, O. Sename, L. Dugard, P. Gaspar, Z. Szabo, J. Bokor, "A new semi-active suspension control strategy through LPV technique", Control Engineering Practice, vol.16, pp.15191534, 2008.

[17] B. Moore, "On the flexibility offered by state feedback in multivariable system beyond closed loop eigenvalue assignment", IEEE Transactions on Automatic Control,vol.21, pp.659692, 1976.

[18] C. Doll, Y. Le Gorrec, G. Ferreres, J.F Magni, "A robust self-scheduled missile autopilot: design by multi-model eigenstructure assignment". Control Engineering Practice, vol.9 pp.1067-1078, 2001.

[19] J.F. Magni, Y. Le Gorrec, C. Chiappa. "An observer based multimodel control design approach". International Journal of Systems Science, pp.6168, 1999.

[20] J.F. Magni, Y. Le Gorrec, C. Chiappa, "An observer based multimodel control design approach", Asian Control Conference, Seoul, 1997. 